\title{
Labyrinthe
}

3 | 1999

Numéro 3

\section{Plaute, Les Bacchides scène 5 vers 235-367}

\section{Florence Dupont}

\section{(2) OpenEdition}

Journals

Édition électronique

URL : http://journals.openedition.org/labyrinthe/68

DOI : $10.4000 /$ labyrinthe.68

ISSN : 1950-6031

Éditeur

Hermann

Édition imprimée

Date de publication : 15 avril 1999

Pagination : 100-113

\section{Référence électronique}

Florence Dupont, "Plaute, Les Bacchides scène 5 vers 235-367 », Labyrinthe [En ligne], 3 | 1999, mis en ligne le 07 février 2005, consulté le 30 avril 2019. URL : http://journals.openedition.org/labyrinthe/68 ; DOI : 10.4000/labyrinthe.68

Ce document a été généré automatiquement le 30 avril 2019

Traduction inédite Florence Dupont, Paris III. Tous droits réservés. 


\title{
Plaute, Les Bacchides scène 5 vers 235-367
}

\author{
Florence Dupont
}

1 Il s'agit d'une scène de ruse et de tromperie, scène attendue par le public, où l'esclave principal, qui est maître du jeu, invente une histoire rocambolesque pour détourner l'argent de Nicobule au profit de son fils Mnésiloque qui en a besoin pour payer sa ruineuse maîtresse, la courtisane Bacchis, et la racheter à un soldat qui a loué ses services pour un an.

2 La scène est parlée, et non chantée ou dansée, et les acteurs n'utilisent que leur voix, sans jouer physiquement leur rôle. C'est donc un spectacle de mots, comme aujourd'hui certains sketchs de music-hall, ceux de Raymond Devos par exemple. D'où l'importance de la traduction. On a choisi, quand c'était nécessaire, de donner des équivalents contemporains aux réalités antiques : par exemple l'Ionie devient la Turquie.

3 Le lecteur reconnaîtra dans cette scène un des modèles probables de la fameuse scène des Fourberies de Scapin, «Qu'allait-il faire dans cette galère ? "

4 NICOBVLVS senex CHRYSALVS seruus

$5 \quad$ NICOBVLVS « Ibo in Piraeum ; uisam ecquaen aduenerit

In portum ex Epheso nauis mercatoria.

Nam meus formidat animus, nostrum tam diu

Ibi desidere neque redire filium.

6 CHRYSALVS Extexam ego illum pulchre iam, si di uolunt.

Haud dormitandumst ; opus est chryso Chrysalo.

Adibo hunc, quem quidem ego hodie faciam hic arietem

Phrixi ; itaque tondebo auro usque ad uiuam cutem.

Seruus salutat Nicobulum Chrysalus.

7 NICOBVLVS Pro di immortales, Chrysale, ubi mist filius ?

8 CHRYSALVS Quin tu salutem primum reddis quam dedi ?

$9 \quad$ NICOBVLVS Salue. Sed ubinamst Mnesilochus? 

Istius hominis ubi fit quomque mentio.

Tum hospitem illum nominas hostem tuum?

\section{Primumdum infitias ire coepit filio,}

Negare se debere tibi triobolum.

Continuo antiquum hospitem nostrum sibi

Mnesilochus aduocauit, Pelagonem senem ;

Eo praesente homini extemplo ostendit symbolum

Quem tute dederas ad eum ut ferret filio.

NICOBVLVS Quid, ubi ei ostendit symbolum?

CHRYSALVS Infit dicere

Adulterinum et non eum esse symbolum;

Quotque innocenti ei dixit contumelias !

Adulterare eum aibat rebus ceteris.

NICOBVLVS Habetin aurum? id mihi dici uolo.

CHRYSALVS Postquam quidem praetor recuperatores dedit, Damnatus demum, ui coactus reddidit Ducentum et mille Philippum.

NICOBVLVS Tantum debuit.

CHRYSALVS Porro etiam ausculta pugnam quam uoluit dare.

NICOBVLVS Etiamnest quid porro?

CHRYSALVS Em, accipetrina haec nunc erit.

NICOBVLVS Deceptus sum; Autolyco hospiti aurum credidi.

CHRYSALVS Quin tu audi.

NICOBVLVS Immo ingenium auidi haud pernoram hospitis. 
Domi cupientes. Forte ut adsedi in stega,

Dum circumspecto, atque ego lembum conspicor

Longum, strigorem, maleficum exornarier.

Occepi ego obseruare eos quam rem gerant.

Interea e portu nostra nauis soluitur.

Vbi portu eximus, homines remigio sequi,

Neque aues neque uenti citius. Quoniam sentio

Quae res gereretur, nauem extemplo statuimus.

Quoniam uident nos stare, occeperunt ratem

Turbare in portu.

41 NICOBVLVS Edepol mortalis malos.

Quid denique agitis?

42

Quoniam uidemus auro insidias fieri,

Capimus consilium continuo ; postridie

Auferimus aurum omne, illis praesentibus,

Palam atque aperte, ut illi id factum sciscerent.

Quoniam extemplo a portu ire nos cum auro uident,

Subducunt lembum capitibus quassantibus.

Nos apud Theotimum omne aurum deposiuimus,

Qui illic sacerdos est Dianae Ephesiae. 

Nimio hic priuatim seruaretur rectius.

Sed nihilne attulistis inde auri domum?

Profecto de auro nihil scio nisi nescio.

Nunc tibimet illuc naui capiundumst iter,

Vt illud reportes aurum ab Theotimo domum.

Atque heus tu!

61

74 NICOBVLVS Censebam me effugisse a uita marituma, Ne nauigarem tandem hoc aetatis senex. Id mi haud utrum uelim licere intellego, 
Ita bellus hospes fecit Archidemides.

Vbi nunc est ergo meus Mnesilochus filius?

Exorsa haec tela non male omnino mihi est,

Ita feci, ut auri quantum uellet sumeret,

Quantum autem lubeat reddere, ut reddat patri.

Senex in Ephesum ibit aurum arcessere ;

Hic nostra agetur aetas in malacum modum,

Siquidem hic relinquet neque secum abducet senex

Med et Mnesilochum. Quas ego hic turbas dabo!

Sed quid futurumst, cum hoc senex resciuerit?

Cum se excucurrisse illuc frustra sciuerit

Nosque aurum abusos, quid mihi fiet postea?

Credo hercle adueniens nomen mutabit mihi

Facietque extemplo Crucisalum me ex Chrusalo.

Aufugero hercle, si magis usus uenerit.

Si ero reprehensus, macto ego illum infortunio ;

Si illi sunt uirgae ruri, at mihi tergum domist. »

Nicobule sort de sa maison pour aller vers le port voir si son fils Mnésiloque est revenu de Turquie. Ledoré, qui vient de débarquer avec lui, le guettait et l'arrête.

NICOBULE, vieux LEDORE, esclave

NICOBULE (aparté). «- Je descends au port pour voir si un navire marchand ne serait pas arrivé de Turquie. J'ai comme un mauvais pressentiment : pourquoi mon fils s'attarde-t-il si longtemps là-bas, pourquoi ne rentre-t-il pas?

LEDORÉ (aparté). - Je vais vous le détricoter gaiement, si les dieux sont avec moi. Ce n'est pas le moment de dormir, Ledoré il te faut de l'or.

Je vais engager la conversation : à l'attaque, il sera mon Jason, l'hom-me à la Toison d'or et je vais lui tondre la laine sur le dos, jusqu'au dernier poil doré.

Ledoré, esclave, salue son maître Nicobule.

NICOBULE. - Grands dieux, Ledoré ! Où est mon fils ?

LEDORÉ. - Comment? Je te salue et tu ne me réponds pas?

NICOBULE. - Salut Ledoré ! mais où est donc Mnésiloque?

LEDORÉ. - Il est en vie. Il va bien.

NICOBULE. - Il ne vient pas?

LEDORÉ. - Il arrive.

NICOBULE. - Ouf, tu m'as rendu la vie. Il n'a pas été malade au moins ?

LEDORÉ. — Lui ? Une santé de fer ! Un athlète ! Un boxeur !

NICOBULE. - Et qu'en est-il de l'affaire que je lui avais confiée et pourlaquelle il était parti en Turquie ? Il a bien récupéré l'or que me devait monami Archidémide? 

ami ! crapule... ? roi des voleurs. pirates...

LEDORÉ. - Hélas ! (hurlement) J'ai le coeur qui se fend, la tête qui éclate quand on prononce son nom devant moi, ce salopard! Quand je pense que tu viens de l'appeler ton

NICOBULE. - Comment? Parle, bon Dieu, je t'en prie !

LEDORÉ. - Dieux tout puissants, s'il est une chose dont je suis sûr, c'est que ni le Soleil ni la Lune, ni le Jour ni le Feu, jamais ce quarteron divin n'a brillé pour une plus abominable

NICOBULE. - Archidémide?

LEDORÉ. - Archidémide. Parfaitement.

NICOBULE. - Mais qu'a-t-il fait?

LEDORÉ. - Que n'a-t-il pas fait, plutôt, c'est ce que tu devrais me demander. D'abord il a commencé par contester la dette, il a prétendu à ton fils qu'il ne te devait pas un sou. Mais Mnésiloque a pris à témoin un ancien ami à vous, le vieux Pélagon. Et en présence de ce Pélagon, il a produit à ton bonhomme la reconnaissance de dette que tu lui avais confiée au moment de son départ.

NICOBULE. - Et alors qu'a-t-il fait quand mon fils lui a montré la reconnaissance de dette

LEDORÉ. - Il a dit que c'était un faux et qu'il ne l'avait jamais vue auparavant. Il l'a traité de faussaire, d'escroc et autres noms d'oiseaux...

NICOBULE. - Avez-vous oui ou non récupéré la somme ? C'est ce qui m'intéresse.

LEDORÉ. - Attends ! Le président du tribunal local, après l'avoir condamné, nous a bien attribué des récupérateurs. Il a été forcé de nous payer les douze cents pièces d'or...

NICOBULE. - C'est exactement ce qu'il devait.

LEDORÉ - Oui mais attends un peu ! Il a monté un coup.

NICOBULE. - Ce n'est pas fini ? Qu'est-ce qui s'est passé encore?

LEDORÉ. - Il a monté un coup tordu, tu vas voir.

NICOBULE. - Je me suis vraiment fait avoir. J'ai cru confier mon or à un ami et c'était le

LEDORÉ. - Tu m'écoutes?

NICOBULE. - Non, je ne connaissais pas son caractère cupide...

LEDORÉ. - Donc, nous avons emporté l'or et nous nous sommes embarqués, ayant très envie de rentrer à la maison. Il se trouve que je me reposais sur le pont, regardant le port autour de moi, quand soudain j'aperçois un canot, long et pointu, avec une sale mine, autour duquel on s'affairait pour l'armer.

11 NICOBULE. - Cette fois, c'est la fin. Ce canot me donne le mal de mer.

LEDORÉ. - Le canot appartenait en commun à notre ami Archidémide et à une bande de

3 NICOBULE. - Il faut être né de la dernière pluie pour faire confiance à un type qui s'appelle Archidémide. C'était écrit qu'il allait m'archidé-pouiller si je lui prêtais quoi que ce soit à cet archivoleur !

4 LEDORÉ. - Le canot avait le projet d'aborder notre navire par surprise. Je me mis à guetter leurs façons de faire. Pendant que notre navire appareille pour quitter le port, ils 
nous suivent à la rame, ils filaient comme le vent, des oiseaux de mer ! Comme je comprends leur manège, aussitôt je fais mettre en panne. Et eux comme ils voient que nous n'avançons plus, ils font des ronds dans le port.

NICOBULE. - Grands dieux, les salopards ! Et vous qu'avez-vous fait finalement?

LEDORÉ. - Nous sommes rentrés.

NICOBULE. - Vous avez agi sagement. Et eux ?

LEDORÉ. - Eux, ils sont revenus à terre, le soir tombé.

NICOBULE. - C'est sûr, ils voulaient s'emparer de l'or. Voilà la seule explication à leur sortie en mer.

LEDORÉ. - Cela ne m'a pas échappé, moi aussi je l'ai compris ainsi. J'étais désespéré. Mais comme nous voyons qu'ils veulent nous voler l'or, nous changeons sur le champ de programme et le lendemain nous sortons tout l'or du navire, sous leurs yeux, ouvertement, ostensiblement, pour qu'ils soient bien au courant de la situation.

NICOBULE. - Bien. Mais, dis-moi, et eux ?

LEDORÉ. - Ils faisaient la pâle gueule et dès qu'ils voient que nous emportons l'or et quittons le port, ils tirent le canot à terre en s'arrachant les cheveux. Quant à nous, nous avons déposé l'or chez Théotime, le grand prêtre de Diane, à Éphèse.

NICOBULE. - Qui est ce Théotime?

LEDORÉ. - Le fils de Mégalobule, l'homme le plus populaire d'Éphèse, là-bas les gens l'adorent.

NICOBULE. - Pourvu que lui n'adore pas trop mon or et qu'il n'aille pas le détourner à son tour.

LEDORÉ. - Comment veux-tu ? L'or est enfermé dans le temple de Diane, un bâtiment public, et là, il est sous la protection de l'État.

NICOBULE. - Tu me tues. Il serait bien mieux, ici, privatisé et sous ma protection. Mais vous n'avez donc rien rapporté ici?

LEDORÉ. - Si, nous en avons rapporté un peu, mais combien, je ne sais pas.

NICOBULE. - Comment, tu ne sais pas?

LEDORÉ. - Parce que Mnésiloque est allé secrètement une nuit chez Théotime ; il n'a rien dit à personne, ni à moi ni à quiconque de l'équipage, c'est pourquoi j'ignore ce qu'il a emporté ; mais ce n'est pas grand chose.

NICOBULE. - La moitié, tu crois?

LEDORÉ. - Je ne sais pas. La moitié ?... Non, sûrement pas.

NICOBULE. - Le tiers, alors ? Il a bien rapporté le tiers de la somme?

LEDORÉ. - Non, je ne crois pas. Mais... mais... non, je ne sais pas. Assurément, pour tout dire, de cet or, je ne sais qu'une chose, c'est que je ne sais rien. Maintenant, je pense qu'il faut que tu t'embarques et que ce soit toi qui ailles chercher l'or chez Théotime pour le rapporter à la maison. Holà ! Attends !

NICOBULE. - Quoi ? Que veux-tu?

LEDORÉ. - L'anneau de ton fils, n'oublie pas de l'emporter !

NICOBULE. - Son anneau ? Pour quoi faire? 
LEDORÉ. - Parce que c'est le signe convenu avec Théotime ; il donnera l'or à celui qui aura cet anneau avec lui.

NICOBULE. - Je n'oublierai pas. Tu as bien fait de me prévenir. Mais ce Théotime, il est riche?

LEDORÉ. - Tu me demandes s'il est riche ? Il s'essuie les pieds sur des paillassons dorés.

NICOBULE. - Pourquoi tant de mépris pour l'or?

LEDORÉ. - Il est si riche, il ne sait plus qu'en faire.

NICOBULE. - Je préférerais qu'il me le donne. Mais qui était présent quand on a remis l'or à Théotime?

LEDORÉ. - Le peuple était là ; tout le monde à Éphèse est au courant.

NICOBULE. - Sur ce point-là du moins mon fils a agi sagement. Il a bien fait de confier l'or en dépôt chez un homme riche. Il nous laissera le reprendre dès qu'on voudra.

LEDORÉ. - Absolument, il ne te fera pas attendre ; dès ton arrivée tu auras la somme.

NICOBULE. - Je croyais en avoir fini avec les voyages en bateau, naviguer à mon âge ! Mais j'ai bien compris que je n'avais pas le choix. Le joli coco que mon ami Archidémide !

À l'heure qu'il est, où est mon fils Mnésiloque?

LEDORÉ. - Il est allé au forum, présenter ses devoirs aux dieux et à ses amis.

NICOBULE. - Moi aussi j'y vais, pour le rejoindre au plus vite. (Il sort)

LEDORÉ. - Je l'ai complètement entortillé dans mes histoires et il n'est pas près de s'en dépêtrer. Il faut dire que mon intrigue n'est pas mal tissée. Le fils de mon maître a de quoi maintenant entretenir ses amours. Il va avoir de l'or à la pelle. Il ne rendra à son père que ce qu'il voudra bien lui rendre. Quant au vieux il va partir à Éphèse pour récupérer son or. Et nous ici, on se la coulera douce, si du moins il nous laisse ici et n'imagine pas de nous emmener avec lui, Mnésiloque et moi. On va faire une de ces javas !

2 Mais que se passera-t-il quand le vieux rentrera ? Quand il se sera aperçu qu'il était parti en Turquie pour rien et que nous avons dépensé l'or, que m'arrivera-t-il à moi ? Je crois qu'à son retour il me fera changer de nom et que Ledoré va devenir Lécorché. Je m'enfuirai si c'est la seule solution. Et si je suis repris, je le maudirai par tous les diables. Il peut élever des martinets dans ses fermes, ici, en ville on a des tanneurs qui nous font des dos durs comme de la corne.

Maintenant je vais y aller, pour donner le mode d'emploi de cette histoire d'or au fils de mon maitre et lui apprendre que sa maîtresse Bacchis est retrouvée. »

\section{AUTEUR}

FLORENCE DUPONT

Florence DUPONT est Professeur à Paris VII et à Paris III. Chercheur au Centre Louis Gernet (URA 884), elle est Directeur de programme au Collège International de 
Philosophie. Elle a traduit notamment Le Théâtre de Sénèque (2 vol.), Imprimerie nationale, 1990-1991. 